PROCEEDINGS OF THE AMERICAN MATHEMATICAL SOCIETY

Volume 126, Number 3, March 1998, Pages 809-814

S 0002-9939(98)04312-3

\title{
PSEUDO-UNIFORM CONVERGENCE, A NONSTANDARD TREATMENT
}

\author{
NADER VAKIL
}

(Communicated by Andreas R. Blass)

\begin{abstract}
We introduce and study the notion of pseudo-uniform convergence which is a weaker variant of quasi-uniform convergence. Applications include the following nonstandard characterization of weak convergence. Let $X$ be an infinite set, $B(X)$ the Banach space of all bounded real-valued functions on $X, \quad\left\{f_{n}: n \in N\right\}$ a bounded sequence in $B(X)$, and $f \in B(X)$. Then the sequence converges weakly to $f$ if and only if the convergence is pointwise on $X$ and, for each strictly increasing function $\sigma: N \rightarrow N$, each $x \in{ }^{*} X$, and each $n \in{ }^{*} N_{\infty}$, there is an unlimited $m \leq n$ such that ${ }^{*} f * \sigma(m)(x) \simeq{ }^{*} f(x)$.
\end{abstract}

Let $f_{\alpha}: X \rightarrow R(\alpha \in \Lambda)$ be a net converging pointwise to $f: X \rightarrow R$. Recall that the convergence is called quasi-uniform on $X$ if we also have:

(A) for each $\epsilon \in R^{+}$and each $\alpha \in \Lambda$, there are $\beta_{1}, \ldots, \beta_{n} \geq \alpha$ such that for each $x \in X, \min _{1 \leq i \leq n}\left|f_{\beta_{i}}(x)-f(x)\right| \leq \epsilon$.

The convergence is called almost uniform if every subnet of $f_{\alpha}: X \rightarrow R$ converges quasi-uniformly to $f: X \rightarrow R$.

Quasi-uniform and almost uniform convergence have interesting applications in functional analysis for questions of weak topology. See, for example, [D-S] page 268, and the works of G. Sirvint [S], R. G. Bartle [B], and J. W. Brace [Br].

A nonstandard formulation of quasi-uniform convergence may be stated in terms of the following notation: for each $\alpha \in{ }^{*} \Lambda$ and each subset $K$ of $X$, set $K_{\alpha}=$ $\left\{x \in{ }^{*} K:{ }^{*} f_{\alpha}(x) \simeq{ }^{*} f(x)\right\}$. Also recall that the members of the set ${ }^{*} \Lambda_{\infty}=$ $\left\{\mu \in{ }^{*} \Lambda: \mu \geq \alpha\right.$ for all $\left.\alpha \in \Lambda\right\}$ are called unlimited.

It is not difficult to prove that Condition (A) above (replacing $X$ with $K$ ) is equivalent to the nonstandard condition:

$$
{ }^{*} K=\bigcup_{1 \leq i \leq \omega} K_{\alpha_{i}} \text { for some hyperfinite set of unlimited indices } \alpha_{1}, \ldots, \alpha_{\omega} .
$$

We replace quasi-uniform convergence with a weaker variant and show that we can still derive results similar to those obtainable from quasi-uniform convergence.

\section{Pseudo-Uniform Convergence}

This section consists of the definition, nonstandard formulation, and some of the basic properties of pseudo-uniform convergence.

Received by the editors October 31, 1995 and, in revised form, September 6, 1996.

1991 Mathematics Subject Classification. Primary 46S20, 03 H05.

(C)1998 American Mathematical Society 
(1.1) Definition. Let $X$ be an infinite set and $f_{\alpha}: X \rightarrow R(\alpha \in \Lambda)$ a net of functions converging to $f: X \rightarrow R$ pointwise on $X$. We call the convergence pseudo-uniform on $X$ if, for each $\epsilon \in R^{+}$and each $\alpha \in \Lambda$, there is $\beta \geq \alpha$ such that for each $x \in X$

$$
\inf \left\{\left|f_{\nu}(x)-f(x)\right|: \alpha \leq \nu \leq \beta\right\}<\epsilon .
$$

The convergence is called topologically pseudo-uniform if every subnet of $f_{\alpha}: X \rightarrow$ $R$ converges pseudo-uniformly to $f: X \rightarrow R$.

Remark. Pseudo-uniform convergence, though a weaker notion than quasi-uniform convergence, behaves in a manner similar to the latter in many applications including the ones described in this paper. Part of the reason for this similarity is the fact that the two notions are equivalent for a large class of nets $\left\{f_{\alpha}: \alpha \in \Lambda\right\}$ for which the directed set $\Lambda$ has the property that, for each $\alpha, \beta \in \Lambda$, the interval $[\alpha, \beta]$ has finite cardinality. For convenience, we shall call such nets interval-finite.

The class of interval-finite nets, of course, includes all sequences. Such nets also arise in integration theory where the directed set is the set of all finite subsets of some infinite set, and the order relation is inclusion.

We observe further that if $(X, \tau)$ is a topological space, $E \subset X$, and $x \in X$, then $x \in \bar{E}$ (the closure of $E$ ) if and only if there is an interval-finite net in $E$ that converges to $x$. To prove this, suppose $x \in \bar{E}$. Let $\mathcal{S}$ be a subbase for the neighborhood system at $x$. Let $\Lambda$ be the set of all finite subsets of $\mathcal{S}$ ordered by inclusion. For each $\alpha \in \Lambda$, pick a point $x_{\alpha}$ in the intersection of $\cap \alpha$ and $E$. Then $\left\{x_{\alpha}: \alpha \in \Lambda\right\}$ is an interval-finite net in $E$ that converges to $x$. The other direction is obvious.

We have noted that quasi-uniform convergence implies pseudo-uniform convergence in general. Clearly, the same implication holds, in general, between almost uniform and topologically pseudo-uniform convergence. Our observation in the above paragraph gives some indication of the fact that more can be said about the relationship between these concepts. We shall, however, attend to a further study of this relationship in a different paper.

We proceed to investigate the properties of pseudo-uniform convergence using the nonstandard language. We assume that the reader is familiar with the elements of nonstandard analysis as treated, for example, in [H-L]. The nonstandard model used in this paper is assumed to be sufficiently saturated for our needs.

(1.2) Theorem. Let $X$ be an infinite set and $f_{\alpha}: X \rightarrow R(\alpha \in \Lambda)$ a net of functions converging to $f: X \rightarrow R$ pointwise on $X$. The convergence is pseudouniform if and only if for each $x \in{ }^{*} X$ and each $\mu \in{ }^{*} \Lambda_{\infty}$, there is an unlimited $\nu \leq \mu$ such that ${ }^{*} f_{\nu}(x) \simeq{ }^{*} f(x)$

Proof. Assume that convergence is pseudo-uniform. Fix $\mu \in{ }^{*} \Lambda_{\infty}$ and $x \in{ }^{*} X$. For each $\alpha \in \Lambda$ and each $\epsilon \in R^{+}$, by hypothesis, the internal sets

$$
E(\alpha, \epsilon)=\left\{\nu \in{ }^{*} \Lambda: \alpha \leq \nu \leq \mu \text { and }\left|{ }^{*} f_{\nu}(x)-{ }^{*} f(x)\right| \leq \epsilon\right\}
$$

are non-empty and have the finite intersection property. Hence there is $\nu \in{ }^{*} \Lambda_{\infty}$ that belongs to $E(\alpha, \epsilon)$ for all $\alpha \in \Lambda$ and all $\epsilon \in R^{+}$. That is, there is $\nu \in{ }^{*} \Lambda_{\infty}$ such that $\nu \leq \mu$ and ${ }^{*} f_{\nu}(x) \simeq{ }^{*} f(x)$.

Conversely, fix $\alpha \in \Lambda$ and $\epsilon \in R^{+}$. Choose any $\beta \in{ }^{*} \Lambda_{\infty}$. Given $x \in{ }^{*} X$, by hypothesis, there is $\nu \in{ }^{*} \Lambda_{\infty}$ such that $\nu \leq \beta$ and $\left|{ }^{*} f_{\nu}(x)-{ }^{*} f(x)\right| \leq \epsilon$. 
Thus we have shown

$$
\left(\exists \beta \in{ }^{*} \Lambda\right)\left(\forall x \in{ }^{*} X\right)\left(\exists \nu \in{ }^{*} \Lambda\right)\left[\alpha \leq \nu \leq \beta \text { and }\left|{ }^{*} f_{\nu}(x)-{ }^{*} f(x)\right| \leq \epsilon\right] .
$$

Now apply the transfer principle to obtain the desired condition for pseudo-uniform convergence.

We now show that, for a net of continuous functions, pseudo-uniform convergence is a necessary condition for continuity of its pointwise limit.

(1.3) Theorem. Let $X$ be a topological space and $f_{\alpha}: X \rightarrow R(\alpha \in \Lambda)$ a net of continuous functions converging pointwise on $X$ to a function $f: X \rightarrow R$. If $f$ is continuous on $X$, then the convergence is pseudo-uniform on every compact subset $K$ of $X$.

Proof. Let $K$ be any compact subset of $X$. Fix $\mu \in{ }^{*} \Lambda_{\infty}$ and $x \in{ }^{*} K$. Given $\epsilon \in R^{+}$and $\alpha \in \Lambda$, consider the internal set

$$
E(\alpha, \epsilon)=\left\{\nu \in{ }^{*} \Lambda: \alpha \leq \nu \leq \mu \text { and }\left|{ }^{*} f_{\nu}(x)-{ }^{*} f_{\nu}\left({ }^{\circ} x\right)\right| \leq \epsilon\right\} .
$$

Since, for each standard $\nu$, the function $f_{\nu}$ is continuous at ${ }^{\circ} x$, the sets $E(\alpha, \epsilon)$ are non-empty and have the finite intersection property. By saturation, it follows that there is an unlimited $\nu \leq \mu$ such that ${ }^{*} f_{\nu}(x) \simeq{ }^{*} f_{\nu}\left({ }^{\circ} x\right)$. Since we also have ${ }^{*} f_{\nu}\left({ }^{\circ} x\right) \simeq{ }^{*} f\left({ }^{\circ} x\right)$ and ${ }^{*} f(x) \simeq{ }^{*} f\left({ }^{\circ} x\right)$, the desired result follows.

As an application of the above results, we obtain a nonstandard proof of Dini's classical theorem for nets.

(1.4) Theorem. Let $X$ be a topological space and $f_{\alpha}: X \rightarrow R(\alpha \in \Lambda)$ a net of continuous functions converging pointwise on $X$ to a continuous function $f: X \rightarrow R$. If the net is monotone, then the convergence is uniform on every compact subset $K$ of $X$.

Proof. Suppose the net is monotone increasing. Fix $x \in{ }^{*} K$ and $\mu \in{ }^{*} \Lambda_{\infty}$. Since $f$ is continuous, by Theorem (1.3), there is $\nu \in{ }^{*} \Lambda_{\infty}$ such that $\nu \leq \mu$ and ${ }^{*} f_{\nu}(x) \simeq{ }^{*} f(x)$. Since ${ }^{*} f_{\nu}(x) \leq{ }^{*} f_{\mu}(x) \leq{ }^{*} f(x)$, it follows that ${ }^{*} f_{\mu}(x) \simeq{ }^{*} f(x)$ as desired.

\section{PARTial EQUicontinuity}

It is well known that quasi-uniform convergence is sufficient for continuity of the pointwise limit of a net $f_{\alpha}: X \rightarrow R(\alpha \in \Lambda)$ of continuous functions. Thus the same is true of pseudo-uniform convergence provided the net is interval-finite. The role of finiteness of the intervals $[\alpha, \beta](\alpha, \beta \in \Lambda)$ in the proof of sufficiency is that of ensuring the equicontinuity of the sets $\left\{f_{\nu}: \alpha \leq \nu \leq \beta\right\}$ for all $\alpha, \beta \in \Lambda$. This partial equicontinuity requirement can be weakened in the following notion.

(2.1) Definition. Let $(X, \tau)$ be a topological space, $p \in X$, and $\tau_{p}=\{V \in \tau$ : $p \in V\}$. A net $f_{\alpha}: X \rightarrow R(\alpha \in \Lambda)$ is called partially equicontinuous at $p$ if, for each $\epsilon \in R^{+}$and each $\alpha \in \Lambda$, there are $\beta \in \Lambda$ and $V \in \tau_{p}$ such that

$$
\inf \left\{\sup _{\beta \leq \nu \leq \mu}\left|f_{\nu}(x)-f_{\nu}(p)\right|: \alpha \leq \mu\right\} \leq \epsilon \text { for all } x \in V .
$$


Remark. Any interval-finite net $f_{\alpha}: X \rightarrow R(\alpha \in \Lambda)$ of continuous functions is partially equicontinuous at all points $p \in X$. To see this, fix $\epsilon \in R^{+}$and $\alpha \in \Lambda$. Pick any $\beta \in \Lambda$ and set

$$
V=\bigcup_{\alpha \leq \mu}\left\{x \in X:\left|f_{\nu}(x)-f_{\nu}(p)\right|<\epsilon \text { for all } \nu \in[\beta, \mu]\right\} .
$$

Partial equicontinuity is the requirement that the set $V$ defined above be a neighborhood of the point $p$. Since each interval $[\beta, \mu]$ has finite cardinality, and, for each $\nu$, the function $f_{\nu}$ is continuous at $p$, the set $V$ belongs to $\tau_{p}$; and it clearly contains $p$.

In the next theorem we provide a simple nonstandard characterization of partial equicontinuity.

(2.2) Theorem. A net $\left\{f_{\alpha}: \alpha \in \Lambda\right\}$ of real-valued functions on $X$ is partially equicontinuous at $p \in X$ if and only if for each $x \simeq p$, there is $\mu \in{ }^{*} \Lambda_{\infty}$ such that ${ }^{*} f_{\nu}(x) \simeq{ }^{*} f_{\nu}(p)$ for all unlimited $\nu \leq \mu$.

Proof. Suppose the net is partially equicontinuous at $p$, and fix $x \simeq p$. Given $\epsilon \in R^{+}$and $\alpha \in \Lambda$, choose $\beta \in \Lambda$ and $V \in \tau_{p}$ as provided by the hypothesis, and set

$E(\alpha, \epsilon)=\left\{\mu \in{ }^{*} \Lambda: \mu \geq \alpha\right.$ and $\left.\left(\forall \nu \in{ }^{*} \Lambda\right)\left[\beta \leq \nu \leq \mu \longrightarrow\left|{ }^{*} f_{\nu}(x)-{ }^{*} f_{\nu}(p)\right|<\epsilon\right]\right\}$.

Since $x \in{ }^{*} V$, it follows from the hypothesis that these internal sets are nonempty. It is also clear that these sets have the finite intersection property. Hence, by saturation, there is an unlimited $\mu \in{ }^{*} \Lambda$ that satisfies the required property.

Conversely, assume that the given nonstandard condition holds. Fix $\epsilon \in R^{+}$and $\alpha \in \Lambda$. Choose any $\beta \in{ }^{*} \Lambda_{\infty}$, and any $V \in{ }^{*} \tau_{p}$ that is contained in the monad of $p$. Given $x \in V$, by hypothesis, there is $\mu \in{ }^{*} \Lambda_{\infty}$, such that $\left|{ }^{*} f_{\nu}(x)-{ }^{*} f_{\nu}(p)\right|<\epsilon$ for all unlimited $\nu \leq \mu$. Thus we have shown that

$$
\begin{aligned}
\left(\exists \beta \in{ }^{*} \Lambda\right)\left(\exists V \in{ }^{*} \tau_{p}\right)(\forall x \in V)\left(\exists \mu \in{ }^{*} \Lambda_{\alpha}\right)\left(\forall \nu \in{ }^{*} \Lambda\right) & \\
& {\left[\beta \leq \nu \leq \mu \longrightarrow\left|{ }^{*} f_{\nu}(x)-{ }^{*} f_{\nu}(p)\right|<\epsilon\right], }
\end{aligned}
$$

where $\Lambda_{\alpha}=\{\mu \in \Lambda: \alpha \leq \mu\}$. Partial equicontinuity at $p$ follows by transfer.

The following theorem implies that pseudo-uniform convergence is sufficient for continuity of the pointwise limit of an interval-finite net of continuous functions.

(2.3) Theorem. Let $f_{\alpha}: X \rightarrow R(\alpha \in \Lambda)$ be a net of continuous functions converging to $f: X \rightarrow R$ pseudo-uniformly on $X$. Then $f$ is continuous at each point $p \in X$ where the net is partially equicontinuous.

Proof. Fix $x \simeq p$. By Theorem (2.2), we can choose an element $\mu(x) \in{ }^{*} \Lambda_{\infty}$ such that ${ }^{*} f_{\nu}(x) \simeq{ }^{*} f_{\nu}(p)$ for all unlimited $\nu \leq \mu$. For this $x$ and $\mu$, by Theorem (1.2), we find an unlimited $\nu_{0} \leq \mu$ such that ${ }^{*} f_{\nu_{0}}(x) \simeq{ }^{*} f(x)$. From these and ${ }^{*} f_{\nu_{0}}(p) \simeq{ }^{*} f(p)$, we get ${ }^{*} f(x) \simeq{ }^{*} f(p)$ as desired.

The next theorem provides a relationship between pointwise convergence of a net of continuous functions on a compact set $X$ and its pseudo-uniform convergence on a dense subset of $X$. A similar theorem for sequences is proved in [B]. 
(2.4) Theorem. Let $f_{\alpha}: X \rightarrow R(\alpha \in \Lambda)$ be a partially equicontinuous net of continuous functions converging pointwise to a continuous function $f: X \rightarrow R$ on a dense subset $D$ of a compact space $X$. Then the net $\left\{f_{\alpha}: \alpha \in \Lambda\right\}$ converges to $f$ at every point of $X$ if and only if every subnet of $\left\{f_{\alpha}: \alpha \in \Lambda\right\}$ converges to $f$ pseudo-uniformly on $D$.

Proof. The necessity of the condition easily follows from Theorem (1.3). We prove the sufficiency by contradiction. Suppose there is a point $p \in X$, an unlimited $\mu \in{ }^{*} \Lambda$, and a positive number $\epsilon$ such that $\left|{ }^{*} f_{\mu}(p)-{ }^{*} f(p)\right|>\epsilon$. Choose $x \in{ }^{*} D$ such that $x \simeq p$, and let $\Gamma=\left\{\beta \in \Lambda:\left|f_{\beta}(p)-f(p)\right|>\epsilon\right\}$. Since ${ }^{*} \Gamma$ contains an unlimited $\mu \in{ }^{*} \Lambda$, the set $\Gamma$ is cofinal in $\Lambda$. Hence $\left\{f_{\beta}: \beta \in \Gamma\right\}$ is a subnet of $\left\{f_{\alpha}: \alpha \in \Lambda\right\}$. By Theorem (2.2), there is $\mu_{0} \in{ }^{*} \Lambda$, such that (1) ${ }^{*} f_{\nu}(x) \simeq{ }^{*} f_{\nu}(p)$ for all unlimited $\nu \leq \mu_{0}$. Since $\left\{f_{\beta}: \beta \in \Gamma\right\}$ converges to $f$ pseudo-uniformly on $D$, there is an unlimited $\gamma \in{ }^{*} \Gamma$ such that $\gamma \leq \mu_{0}$ and ${ }^{*} f_{\gamma}(x) \simeq{ }^{*} f(x)$. From this, (1), and the continuity of $f$ at $p$, we get ${ }^{*} f_{\gamma}(p) \simeq{ }^{*} f(p)$, which is a contradiction.

\section{Nonstandard CHARACTERIZATION OF WEAK CONVERGENCE}

We first fix the notation to be used in this section. Let $X$ be an infinite set and $B(X)$ the Banach space of all bounded real-valued functions on $X$ (with the supremum norm). For each $f \in B(X)$, let $d_{f}$ denote the pseudometric on $X$ defined by $d_{f}(x, y)=|f(x)-f(y)|$, and let $\mathcal{D}=\left\{d_{f}: f \in B(X)\right\}$. Let $\hat{X}$ be the nonstandard hull of $X$ with respect to the uniformity on $X$ that is generated by $\mathcal{D}$, and let $\hat{f}: \hat{X} \rightarrow R$ be the hull of $f$ (that is, $\hat{f}(\hat{x})=s t\left({ }^{*} f(x)\right)$ ). Recall that $\hat{X}$ is the Stone-Čech compactification of $(X, \mathcal{D})$ (see $[\mathrm{L}]$, page 82 ), and that the set $C(\hat{X})$ of all real-valued continuous functions on $\hat{X}$ equals $\{\hat{f}: f \in B(X)\}$. Also note that, for all $f \in B(X)$, we have $\sup \{|\hat{f}(\hat{x})|: \hat{x} \in \hat{X}\}=\sup \{|f(x)|: x \in X\}$, and that, for all $f, g \in B(X)$ and $r, s \in R$, we have $(r \widehat{f+s} g)=r \hat{f}+s \hat{g}$. That is, the map $f \in B(X) \longmapsto \hat{f} \in C(\hat{X})$ is an isometric and isomorphic bijection between $B(X)$ and $C(\hat{X})$.

To prove our nonstandard characterization of weak-convergence, we need the following result which may be viewed as a nonstandard version of Theorem (2.4) for sequences.

(3.1) Theorem. Let $f_{n}: X \rightarrow R(n \in N)$ be a sequence of continuous functions converging pointwise to a continuous function $f: X \rightarrow R$ on a dense subset $D$ of a compact space $X$. Then the sequence $\left\{f_{n}: n \in N\right\}$ converges to $f$ at every point of $X$ if and only if for each strictly increasing function $\sigma: N \rightarrow N$, each $x \in{ }^{*} D$, and each $n \in{ }^{*} N_{\infty}$, there is an unlimited $m \leq n$ such that ${ }^{*} f{ }^{*} \sigma(m)(x) \simeq{ }^{*} f(x)$.

Proof. Suppose $\left\{f_{n}: n \in N\right\}$ converges to $f$ at every point of $X$. Then so does the sequence $\left\{f_{\sigma(n)}: n \in N\right\}$ for each strictly increasing function $\sigma: N \rightarrow N$. Since $f$ is continuous, Theorems (1.3) and (1.2), in that order, applied to the sequence $\left\{f_{\sigma(n)}: n \in N\right\}$ yield the desired result.

We prove the converse by contradiction. Suppose that $\left\{f_{n}: n \in N\right\}$ does not converge to $f$ at some point $p \in X$. Then there are a positive number $\epsilon$ and a subsequence $\left\{f_{\sigma(n)}: n \in N\right\}$ such that $\left|{ }^{*} f{ }^{*} \sigma(n)(p)-{ }^{*} f(p)\right|>\epsilon$ for all $n \in{ }^{*} N$. Choose $x \in{ }^{*} D$ such that $x \simeq p$. Since $\left\{f_{\sigma(n)}: n \in N\right\}$ is partially equicontinuous at $p$, by Theorem (2.2), there is $n_{\circ} \in{ }^{*} N_{\infty}$ such that (1) 


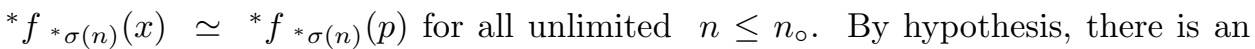
unlimited $m$ such that $m \leq n_{\circ}$ and ${ }^{*} f * \sigma(m)(x) \simeq{ }^{*} f(x)$. From this, continuity of $f$ at $p$, and (1), we get ${ }^{*} f{ }^{*} \sigma(m)(p) \simeq{ }^{*} f(p)$, which is a contradiction.

We are now ready to present the main theorem of the paper.

(3.2) Theorem. A bounded sequence $\left\{f_{n}: n \in N\right\}$ in $B(X)$ converges weakly to $f \in B(X)$ if and only if the convergence is pointwise on $X$ and, for each strictly increasing function $\sigma: N \rightarrow N$, each $x \in{ }^{*} X$, and each $n \in{ }^{*} N_{\infty}$, there is $m \in{ }^{*} N_{\infty}$ such that $m \leq n$ and ${ }^{*} f{ }^{*} \sigma(m)(x) \simeq{ }^{*} f(x)$.

Proof. Suppose $\left\{f_{n}: n \in N\right\}$ converges weakly to $f$. Since, for each $u \in \hat{X}$, the function $\lambda_{u}: B(X) \rightarrow R$ defined by $\lambda_{u}(f)=\hat{f}(u)$ is a bounded functional on $B(X)$, the sequence $\left\{\hat{f}_{n}: n \in N\right\}$ converges pointwise to $\hat{f}$ on $\hat{X}$. Now identify $f_{n}$ with the restriction of $\hat{f}_{n}$ to $X$ and use Theorem (3.1) to obtain the desired condition.

Conversely, by hypothesis and Theorem (3.1), the sequence $\left\{\hat{f}_{n}: n \in N\right\}$ converges to $\hat{f}$ pointwise on $\hat{X}$. From this and boundedness of $\left\{\hat{f}_{n}: n \in\right.$ $N\}$, through Riesz representation and Lebesgue bounded convergence theorems, it follows that $\left\{\hat{f}_{n}: n \in N\right\}$ converges to $\hat{f}$ weakly (see [D-S], p. 265, Corollary 4). Since, for each bounded functional $\lambda$ on $B(X)$, the function $\hat{\lambda}: \hat{B} \rightarrow R$ defined by $\hat{\lambda}(\hat{f})=\lambda f$ is a bounded functional on $\hat{B}$, the weak convergence of $\left\{\hat{f}_{n}: n \in N\right\}$ to $\hat{f}$ implies the weak convergence of $\left\{f_{n}: n \in N\right\}$ to $f$.

\section{ACKNOWLEDGEMENT}

The author is grateful to the annonymous referee and Peter Loeb whose suggestions and comments induced much improvement of the original paper.

\section{REFERENCES}

[B] R. G. Bartle, On compactness in functional analysis, Trans. Amer. Math. Soc. 79, pp. 35-57, 1955. MR 17:510h

[Br] R. W. Brace, Compactness in function spaces, Duke Mathamatical Journal, volume 29, 1962, 157-166. MR 32:4665

[D-S] N. Dunford and J. T. Schwartz, Linear Operators, Part I John Wiley and Sons 1988. MR 90g:47001a

[H-L] A. E. Hurd and P. A. Loeb, An Introduction to Nonstandard Real Analysis, Academic Press 1985. MR 87d:03184

[L] W. A. J. Luxemburg, A general Theory of Monads, Applications of Model Theory to Algebra, Analysis, and Probability (W.A.J. Luxemburg, editor), Holt, Reinhard and Winston, New York, 1969, 18-86. MR 39:6244

[S] G. Sirvint, Weak Compactness in Banach Spaces, Studia Mathematica, vol. 11, pp. 71-94, 1950. MR 14:183c

Department of Mathematics, Western Illinois University, Macomb, Illinois 61455

E-mail address: N-Vakil@bgu.edu 